\title{
NEO fireball diversity: energetics-based entry modeling and analysis techniques
}

\author{
Douglas O. ReVelle
}

\author{
Los Alamos National Laboratory, Los Alamos, New Mexico, USA \\ email: revelle@lanl.gov
}

\begin{abstract}
We have examined the behavior of a number of bolides in Earth's atmosphere from the standpoint of recent entry modeling techniques. The entry modeling has been carried out including a triggered progressive fragmentation model (TPFM) which maintains a maximum drag orientation for the fragments in either the collective or a non-collective wake limit during entry (ReVelle 2004). Specifically in this paper, we have proposed a new method of estimating the terminal bolide mass and have compared it against the corresponding single-body mass loss prediction. A new expression for the terminal mass is proposed that corrects the mass of the body for the changing mass to area ratio during the fragmentation process. As a result of this new work we have found two very interesting features that correspond very closely to those found from a direct analysis of the observational data. These include an instantaneous mass that closely resembles that directly observed and an ablation coefficient behavior that also strongly resembles meteor observations (such as those found recently by Ceplecha \& ReVelle 2005). During fragmentation, the apparent ablation coefficient has now been shown to decrease dramatically approaching the intrinsic ablation coefficient proposed by Ceplecha \& ReVelle (2005). In our modeling we have assumed a breakup into equal size fragments that are consistently and progressively multiples of two of the original unbroken leading piece. Had we assumed a multitude of many much smaller pieces that made up the totality of the original body, our predicted ablation coefficient would indeed have approached the very small intrinsic ablation parameter values predicted by Ceplecha and ReVelle. This is especially evident in the case of Sumava, but is also true in a number of other cases as well. The bolides whose properties have been modeled using our detailed entry code including a prediction of the panchromatic luminosity consist of the 1965 Revelstoke meteorite fall (Folinsbee 1967; Carr 1970; Shoemaker 1983), the 1974 Sumava fireball and the 1991 Benesov fireball as presented in Borovička \& Spurný (1996) and in Borovička et al. (1998), the Tagish Lake meteorite fall of January 8, 2000 (Brown et al. 2002), the March 9, 2002 Park Forest meteorite fall (Brown et al. 2004), the June 6, 2002 Mediterranean (Crete) bolide as presented in Brown et al. (2002) and finally the September 4, 2004 Antarctic bolide respectively (Klekociuk et al. 2005). A self-consistent assessment of the detailed properties of each of the fireballs was made using all available information for each event. In the future, more reliable estimates of all of the necessary source parameters (including their overall degree of bulk porosity) will be made if all channels of information are reliably retrieved for bolide events (channels such as acoustic-gravity waves and specifically its infrasound emission, seismic waves, satellite optical and IR data, ground-based spectroscopy, ground-based photometry and radiometry, VLF radiation, meteorite fragment recovery, etc.).
\end{abstract}

Keywords. meteoroids, fireballs, bolides

\section{Introduction and Overview}

\subsection{Large Fireball Behavior in the Atmosphere}

During the hypersonic continuum flow entry of large meteoroids, the parameters of direct interest are the degree of bolide ablation, the drag and corresponding deceleration, the mass loss and the concomitant degree of fragmentation and the related behavior of the 
fragments in the near-wake region, the panchromatic light production as well as in other spectral bands of interest, an estimation of the total power budget, mechanical wave and radio frequency wave generation processes, possible impact and explosive cratering, etc. In this paper we will examine a number of fireballs, some of which have dropped meteorites and some of which have apparently disintegrated totally during their flight in the atmosphere. The degree to which we are able to understand these events through systematic analyses directly impacts on our ability to understand their possible origins and compositional variability which is very important to our understanding of our solar system and its evolutionary processes. To better understand this complete problem we demand not only entry modeling analyses, but also remote monitoring of the "optical" and acoustic/infrasonic and internal gravity wave production, i.e., acoustic-gravity waves and knowledge of their relationships to the structure and the composition of the meteoroids themselves.

The large range of expected phenomena have made the use of a wide range of observing techniques necessary for a proper understanding of these events and their source energies in order to properly calibrate the expected steady state influx rate of bolides (Brown et al. 2004). Readily available technology for the detection of bolides includes optical and infrared satellite data, ground-based radiometry (as has been developed by R.E. Spalding at Sandia National Laboratory in Albuquerque) and by the detection of acoustic-gravity (mechanical) and seismic waves by the IMS (International Monitoring System) network and by other available infrasonic and seismic sensors/arrays. Additional techniques include ground-based photometry (by the European Fireball Network, etc.), ground-based spectroscopy and detection by VLF radio receivers and of course information gained by direct meteorite recovery efforts, etc.

\subsection{Reliable Prediction of the Terminal Mass of Fireballs}

In previous treatments of entry dynamics (ReVelle 2004), the author has not focused his attention on precise predictions of the bolide's terminal mass. In this work we have provided two estimates of the terminal mass, one based strictly on the standard singlebody theory (with no fragmentation assumed to be present throughout the entry) and a new estimate based upon the self-consistent correction of the mass based upon predicted changes in the mass to area ratio of the bolide during fragmentation. The latter approach reduces uniformly to the single-body predicted limit in the absence of fragmentation. Using this latter approach, significantly smaller values of the terminal mass are now generally predicted under a certain range of entry conditions.

These predictions are also based upon a new evaluation of the mass/area ratio in the non-collective wake behavioral limit. Previous non-collective wake solutions by the author had assumed that the multiplier, $k_{4}$ (see below), for $p^{*}$, the modified ballistic entry parameter (which is proportional to the mass to area ratio of the body) was exactly 1.0 based upon a mass reduction during each specified breaking interval with $k_{3}=0.50$ and a frontal cross-sectional area reduction $k_{2}=0.50$. If however we further demand a maximum drag orientation for the leading fragmented piece as we have also done for the collective wake limit, we now predict that $k_{4}=0.50$ which is identical to that of the collective wake solution (see below for further details). Intermediate solutions are certainly possible for the non-collective wake limit as well up to the limit for $k_{4}=1.0$, but this is in general an unknown detail without further extensive wake calculations. For a value of $k_{4}=0.50$ for both solutions, an identical end height for the two extreme wake behaviors is predicted to occur. For $k_{4}=0.75$ for example, we predict a slightly lower end height for the non-collective wake solution compared to the collective wake solution. For each of these solutions however, we obtain a different prediction of the breaking altitudes 
and the optical luminosity produced as well despite the fact that the end heights are almost identical. Full details of many of these proposed changes will be discussed below.

\section{Entry Modeling Techniques}

\subsection{Single-Body Mass Loss versus Mass Loss with Break-up}

We will start first from the fundamental differential equations of deceleration, mass loss, kinetic energy change, area change, shape factor change and the connection between geopotential altitude, $z$ and time, $t$ that describe the full behavior of the single-body meteor entry assuming negligibly small thermal conduction effects and negligible lift, namely (ReVelle 1979; Bronshten 1983; Ceplecha et al. 1998; ReVelle 2004):

$$
\begin{aligned}
\frac{d V}{d t} & =-0.5 \cdot \rho(z) \cdot V^{2}(z) \cdot C_{D}(z) \cdot\left\{\frac{A(z)}{m(z)}\right\} \\
\frac{d V}{d t} & =-\frac{C_{D}(z) \cdot S_{f}(z) \cdot \rho(z) \cdot V^{2}(z)}{\rho_{m}^{2 / 3} \cdot m^{1 / 3}(z)} \\
\frac{d m(z)}{d t} & =-0.5 \cdot \rho(z) \cdot V^{3}(z) \cdot C_{D}(z) \cdot \sigma(z) \cdot A(z) \\
\frac{d m(z)}{d t} & =-\frac{C_{D}(z) \cdot S_{f}(z) \cdot \sigma(z) \cdot \rho(z) \cdot m^{2 / 3}(z) \cdot V^{3}(z)}{\rho_{m}^{2 / 3}} \\
\frac{d E_{k}(z)}{d t} & =-\frac{I_{\text {pan }}(z)}{\tau_{\text {pan }}(\rho(z), m(z), V(z))} \\
\frac{d A(z)}{d t} & =\mu \cdot\left\{\frac{A(z)}{m(z)}\right\} \cdot \frac{d m(z)}{d t} ; \quad \text { constant } \\
\frac{d S_{f}(z)}{d t} & =-\left[\frac{2}{3}-\mu\right] \cdot S_{f}(z) \cdot\left\{\frac{1}{m(z)} \cdot \frac{d m(z)}{d t}\right\} \\
\frac{d z(t)}{d t} & =-V(z) \cdot \sin \theta ; \quad \theta=\text { constant }
\end{aligned}
$$

where:

- $m(z)=$ instantaneous meteor mass;

- $\theta=$ horizontal entry angle (in the plane parallel earth approximation);

- $C_{D}=$ coefficient of wave drag at hypersonic and supersonic speeds $(\mathcal{O}(1))$;

- $E_{k}=$ kinetic energy of the body;

- $I_{\text {pan }}=$ panchromatic radiation power emission;

- $\tau_{\text {pan }}=$ panchromatic differential luminous efficiency as given in ReVelle (2004);

- $A(z)=$ frontal cross-sectional area of the meteor;

- $V(z)=$ instantaneous meteor velocity;

- $\rho(z)=$ atmospheric density as a function of altitude;

- $\sigma(z)=$ ablation parameter as a function of altitude, $z$;

- $S_{f}(z) \equiv A / V_{0}^{2 / 3}, A(z) / A_{\infty} \equiv\left(m(z) / m_{\infty}\right)^{\mu}$, where $V_{0}=$ meteor volume and $\mu=$ shape change parameter which is assumed constant;

- $S_{f}(z)=$ shape factor;

- $t=$ time of flight along the entry trajectory;

- $z=$ geopotential altitude.

In equation (2.3) above, we can also write numerous similar expressions for the time rate of change of the kinetic energy in terms of the many other forms of kinetic energy deposited by the body into the atmosphere and their corresponding differential efficiencies. 
These include acoustical energy deposition, heat deposition, ionization, dissociation, etc. (ReVelle 2004).

Next we will utilize the modified ballistic entry parameter, $p^{*}(z)$, as one integral part of the overall dynamical entry solution for the case of a constant $\sigma$. For this $p^{*}(z)$ parameter, which is a modified form of the object's mass/area ratio, we have the expression from single-body theory (ReVelle 1979; Bronshten 1983; ReVelle 2004):

$$
p^{*}(z)=p_{\infty}^{*} \cdot e^{-\left[\sigma \cdot \frac{\mu-1}{2}\right] \cdot\left[V_{\infty}^{2}-V^{2}(z)\right]}
$$

where

- $p^{*}(z)=m(z) \cdot g \cdot \sin \theta /\left(C_{D} \cdot A\right)=$ modified ballistic entry parameter;

- $g=$ acceleration due to gravity $(=$ constant $))$, so that changes in $p^{*}$ are proportional to changes in $m / A$ and which is used in equations (2.15)-(2.22) below.

Equation (2.7) may be immediately solved for the instantaneous mass in the form, if $g, C_{D}$ and $\sin \theta$ are assumed constants of the hypersonic aerodynamic motion, i.e., the so-called simple ablation theory:

$$
m(z)=m_{\infty} \cdot\left\{\frac{A(z)}{A_{\infty}}\right\} \cdot e^{\left[\sigma \cdot \frac{\mu-1}{2}\right] \cdot\left[V_{\infty}^{2}-V^{2}(z)\right]} .
$$

Thus, if the cross-sectional area computed previously $\left(A_{\infty}\right)$ is larger than the current cross-sectional area (for collective wake behavior), the quantity in the curly brackets in equation (2.8) is $<1$ with similar expectations for non-collective wake behavior, but with different values for the multiplying constants that are discussed below in Section 2.2. For the condition of maximum drag this area ratio is 0.5 for both the collective and the noncollective wake limits. Using the ancillary single-body cross-sectional area relationship in the form (ReVelle 1979):

$$
A(z)=A_{\infty} \cdot e^{-\left[\sigma \cdot \frac{\mu}{2}\right] \cdot\left[V_{\infty}^{2}-V^{2}(z)\right]}
$$

Equation (2.8) can now be simplified to the standard single-body mass loss form which is completely independent of the shape change parameter as expected (Bronshten 1983):

$$
m(z)=m_{\infty} \cdot e^{-\frac{\sigma}{2} \cdot\left[V_{\infty}^{2}-V^{2}(z)\right]}
$$

or. equivalently. changes in either the single-body radius, $r(z)$, in the frontal crosssectional area, $A(z)$ or in the shape factor, $S_{f}$ with altitude can be expressed in the form:

$$
\begin{aligned}
r(z) & =\left(\frac{S_{f}(z)}{\pi}\right)^{1 / 2} \cdot\left(\frac{m(z)}{\rho_{m}}\right)^{1 / 3} \\
A(z) & =S_{f}(z) \cdot\left(\frac{m(z)}{\rho_{m}}\right)^{2 / 3} \\
S_{f}(z) & =S_{f \infty}\left(\frac{m_{\infty}}{m(z)}\right)^{2 / 3-\mu} .
\end{aligned}
$$

These ancillary equations arise directly from the constant $\sigma$, ballistic entry, analytic solutions for the mass and area loss, etc. Since our entry dynamics solutions are entirely numerical, we must modify these expressions to their form expected during fragmentation as well as express them into a finite difference form for use in our entry dynamics solution 
scheme (which computes detailed entry characteristics for either a constant or a variable $\sigma$ solution for either a hydrostatic, isothermal or non-isothermal atmosphere respectively). In all cases in our initial modeling, we have used $S_{f} \approx 1.209$ which is the value for a sphere.

\subsection{Mass Loss including Fragmentation Effects}

After fragmentation begins, we have modified the surface cross-sectional area and the corresponding heat transfer area (allowing in general for porous meteoroid entry ablation) as well as the mass and modified ballistic entry parameter as follows, depending on whether or not the wake behavior, which is modeled in terms of one of two possible extreme limits, i.e., either a collective or a non-collective wake behavior during entry (with $A=$ drag cross-sectional area and $A_{H}=$ heat transfer area). Thus, triggering of fragmentation is assumed to mechanically occur if:

$$
p_{\text {stag }}(z)>S
$$

where $p_{\text {stag }}(z)=0.5 \cdot \rho(z) \cdot V^{2}(z) \cdot C_{D}(z)$ and $S=$ "Breaking strength"; $S=S($ composition, bulk porosity, etc.) and mechanical breakup is allowed to occur as a cascade process (using our triggered progressive fragmentation model-TPFM)). The value of $S$ is an assigned curve-fitted constant for each bolide type, etc. (ReVelle 2004).

The $k_{i}$ multiplier parameters given below (for $k_{i}, i=1, \ldots 4$ ) have been written with the other parameters in a finite difference form so that the $z-1$ refers to the previous altitude while $z$ refers to the current altitude:

a) Collective wake limit: CWL as specified with a specific time delay after each breakup as a function of the velocity and angle of entry of the bolide, etc.

$$
\begin{aligned}
k_{1} \equiv & \frac{A(z)}{A(z-1)} ; \quad k_{1}>1\left(k_{1}=2.0 \text { nominal }\right) \\
& \text { applied at each specified breaking height } \\
k_{2} \equiv & \frac{A_{H}(z)}{A_{H}(z-1)} ; \quad k_{2}>1(k 2=2.0 \text { nominal }) \\
k_{3} & \equiv \frac{m(z)}{m(z-1)} ; \quad k_{3}=1.0 \\
k_{4} \equiv & \frac{p^{*}(z)}{p^{*}(z-1)} ; \quad k_{4}=\frac{1}{2} .
\end{aligned}
$$

In this CWL limit the broken mass quickly returns toward the leading surviving fragment in the form of "flying buckshot" in a maximum drag orientation while each piece continues to ablate so that the effective area has doubled while the effective mass that participates in the deceleration process has not changed. We have implictly assumed here that the drag coefficent of the swarm of fragments is the same for that of the original unbroken body. The mass is drawn forward because of the heavily decreased air density in the near wake.

b) Non-collective wake limit: NCWL

$$
\begin{aligned}
k_{1} \equiv & \frac{A(z)}{A(z-1)} ; \quad k_{1} \leqslant 1\left(k_{1}=1.0 \text { nominal }\right) \\
& \text { applied at each specified breaking height } \\
k_{2} \equiv & \frac{A_{H}(z)}{A_{H}(z-1)} ; \quad k_{2} \leqslant 1\left(k_{1}=1.0 \text { nominal }\right)
\end{aligned}
$$




$$
\begin{aligned}
k_{3} & \equiv \frac{m(z)}{m(z-1)} ; & k_{3} & =\frac{1}{2} \\
k_{4} & \equiv \frac{p^{*}(z)}{p^{*}(z-1)} ; & k_{4} & =\frac{1}{2} .
\end{aligned}
$$

In this NCWL limit the broken mass is assumed to quickly be "lost" to the far wake and no longer interacts with the leading fragment which continues its flight in a maximum drag orientation while it continues to ablate. Thus, the effective area has remained the same while the effective mass that participates in the deceleration process has been reduced each time by a factor $k_{3}=1 / 2$.

Equations (2.18) and (2.22) imply that the effective $m / A$ ratio has decreased following each of these fragmentation events. Specific values of the above constants determine the possible end height behavior. In the computations, the enhancement of the frontal area is only allowed right at each of the breakup locations which are assumed to be progressive once breakup begins. One physical reason for the continuation of the breakup process after equation (2.14) has been initially satisfied is that for sufficiently small fragments, heat conduction processes can produce thermal shock effects that will be sure to continue the fragmentation process. After the time step where each individual breakup occurs, the $p^{*}$ ratio, etc. is maintained at the original value of the constants (all numerical $k_{i}$ values are set to unity). We list below the key points to be noted for the above range of $k_{i}$ values:

i) The CWL evaluated with $k_{4}=0.50$, has the same end height as for the NCWL evaluated with $k_{4}=0.50$ with all other factors the same. This occurs because the net mass to area ratio is the same for both situations for the case of maximum drag penetration.

ii) The CWL produces optically much brighter entries (due to the greatly increased frontal area of the body in this limit during fragmentation) than does the NCWL with all other factors the same. For Sumava for example, this brightness difference is more than 2 stellar magnitudes (at maximum luminosity the predicted change between these extremes is from $\approx-20$ to -22 ).

The detailed bolide results shown below for these extreme wake behavior limits are determined by the specific set of values listed above even though they are only "turned on" at each of the specific breaking heights in the fragmentation cascade being modeled i.e., in our TPFM model. Each of the limiting wake behavior cases now occurs with nearly identical end heights (for $k_{4}=0.50$ specified for both extreme cases) until the predicted end height velocity was reached.

In addition to the above, there are specific limits to the modified ballistic parameter behavior for either the CWL or NCWL limits. For the former, mass does not change initially and the frontal area increases as the particles rearrange themselves into a maximum drag orientation profile. This causes $p^{*}$ to be reduced to $1 / 2$ briefly at each specified fragmentation height compared to its pre-fragmentation value. In the NCWL, the mass is reduced by a factor of $1 / 2$ and for maximum drag conditions, the drag area remains the same so that the $k_{4}$ is identical to that for the CWL. This not only produces an identical end height for the numerical values specified for the two wake extremes, but also an identical end height velocity (see ReVelle 2004).

In addition, in a future publication the author will explore both the effects of fragmentation on the end height and the luminosity for the case in which the maximum drag orientation can not be achieved. In addition, the future specification of a fragment particle size distribution will also allow a more realistic modeling of the debris field of "flying buckshot" that is envisioned to form during the CWL. 


\subsection{Finite Difference Form of the Resulting Equations}

Equation (2.8) may also be written in a finite difference, iterative entry dynamics solution format:

$$
m(z)=m(z-1) \cdot \frac{A(z)}{A(z-1)} \cdot e^{-\frac{\sigma(z)}{2} \cdot\left[V^{2}(z-1)-V^{2}(z)\right]}
$$

where $z-1$ indicate greater heights than the current altitude, $z$. Since $\sigma$ is lagged to previous heights, so are area and mass values. We have also demanded that

$$
\frac{1}{\sigma(z)} \cdot \frac{\partial \sigma(z)}{\partial z} \cdot \delta z<<1
$$

be satisfied so that any altitude changes are not too severe and only slowly varying.

Prior to fragmentation, Equation (2.10) is just the single-body model ablation equation originating from the drag/deceleration and the mass loss equation where $A(z) / A(z-1)$ is available from Equation (2.9).

i) Prior to and after the termination of progressive fragmentation effects: single-body model

$$
m(z)=m(z-1) \cdot e^{-\frac{\sigma(z)}{2} \cdot\left[V^{2}(z-1)-V^{2}(z)\right]}
$$

ii) After the onset of progressive fragmentation effects:

$$
m(z)=m(z-1) \cdot \frac{A(z)}{A(z-1)} \cdot e^{-\frac{\sigma(z)}{2} \cdot\left[V^{2}(z-1)-V^{2}(z)\right]} .
$$

Equation (2.25) is our proposed correction to the mass loss while accounting for the crosssectional area changes generated by the fragmented bolide. These expressions correct the mass for changes in the $m / A$ ratio during the fragmentation process. In the same way equation (2.10) expresses changes in the single-body mass prior to fragmentation. The advantage of using the $m / A$ approach is that fragmentation influences on the mass loss can be directly incorporated in the modeling process unlike the conventional single-body mass loss equation which only accounts for a quasi-continuous ablation process for a single unbroken body.

Thus, the predicted final meteor masses during fragmentation are extreme lower limits to the "true" mass. As an indicator of the upper mass limit, we have also plotted the single-body model ablation results.

\section{Examples of NEO Fireball Diversity and its Modeling}

\subsection{Benesov/Sumava Fireball Dichotomy: Type I and IIIB Extremes}

In Table 1 we specify the key input parameters such as entry velocity, entry angle, etc. needed for a proper modeling of the drag, deceleration, the corresponding end heights (which in all cases agree quite well with the observations) and the single-body and fragmentation modeled mass loss (using the mass to area ratio approach developed in this paper) as well for modeling of the panchromatic luminosity output for the Benesov and Sumava bolides and for the other five very diverse bolide events presently under consideration. We also list in the same table some of the key outputs resulting from the modeling process such as initial mass, number of fragments produced, terminal velocity at the end height, the required wake behavior model, the necessary bolide type and/or the degree of bulk porosity, etc.

In Figure 1, we have plotted the altitude behavior of panchromatic luminosity, mass loss and $\sigma$ for Benesov and Sumava (Borovivcka \& Spurný 1996; Borovička et al. 1998). 
Table 1. Summary of input modeling parameters assigned for the various bolides (all with $D=4.605$ corresponding to $99 \%$ kinetic energy removal at the end height with $S_{f}=1.209$ for a spherical shape).

\begin{tabular}{|c|c|c|c|c|c|c|}
\hline Bolide & \begin{tabular}{|c} 
Observed \\
$V_{\infty}(\mathrm{km} / \mathrm{s})$ \\
Predicted \\
$V_{t}(\mathrm{~km} / \mathrm{s})$
\end{tabular} & $\begin{array}{c}\text { Predicted } \\
m_{\infty}(\mathrm{kg})\end{array}$ & $\begin{array}{l}\text { Observed } \\
\theta\left({ }^{\circ}\right): \\
\text { Radiant } \\
\text { elevation } \\
\text { angle }\end{array}$ & $\begin{array}{c}\text { Assumed } \\
S_{f} ; \mu ; \\
\text { Limiting wake } \\
\text { model }\end{array}$ & \begin{tabular}{|} 
Predicted \\
bolide type \\
and bulk \\
porosity
\end{tabular} & $\begin{array}{l}\text { Predicted } \\
\text { number of } \\
\text { fragments } \\
\text { for light } \\
\text { curve } \\
\text { modeling }\end{array}$ \\
\hline Sumava & $\begin{array}{l}26.90 \\
25.70\end{array}$ & 3988.6 & 27.5 & $\begin{array}{c}1.209 ; 2 / 3 \\
\text { CWL }\end{array}$ & $\begin{array}{c}\text { IIIA/IIIB } \\
86 \%\end{array}$ & 128 \\
\hline Benesov & $\begin{array}{l}21.18 \\
12.55\end{array}$ & 1550.0 & 80.6 & $\begin{array}{c}1.209 ; 2 / 3 \\
\text { CWL }\end{array}$ & $\begin{array}{c}\text { I } \\
20 \%\end{array}$ & 8 \\
\hline Revelstoke & $\begin{array}{l}13.0 \\
3.25\end{array}$ & $6.54 \cdot 10^{5}$ & 15.0 & $\begin{array}{c}1.209 ; 2 / 3 \\
\text { CWL }\end{array}$ & $\begin{array}{c}\text { II } \\
20 \%\end{array}$ & 16 \\
\hline Tagish Lake & $\begin{array}{l}15.8 \\
9.93\end{array}$ & $1.405 \cdot 10^{5}$ & 18.0 & $\begin{array}{c}1.209 ; 0.10 \\
\text { CWL }\end{array}$ & $\begin{array}{c}\text { II/IIIA } \\
60 \%\end{array}$ & 4 \\
\hline Park Forest & $\begin{array}{c}20.0 \\
10.98\end{array}$ & 3405.0 & 61.0 & $\begin{array}{c}1.209 ; 2 / 3 \\
\text { NCWL }\end{array}$ & $\begin{array}{c}\text { I } \\
20 \%\end{array}$ & 128 \\
\hline Crete bolide & $\begin{array}{l}13.5 \\
3.38\end{array}$ & $7.160 \cdot 10^{5}$ & 60.0 & $\begin{array}{l}1.209 ; 0.05 \\
\text { CWL }\end{array}$ & $\begin{array}{l}\text { I } \\
5 \%\end{array}$ & 8 \\
\hline Antarctic bolide & $\begin{array}{l}13.0 \\
4.99\end{array}$ & $1.237 \cdot 10^{6}$ & 41.9 & $\begin{array}{c}1.209 ; 2 / 3 \\
\text { NCWL }\end{array}$ & $\begin{array}{l}\mathrm{I} / \mathrm{II} \\
50 \%\end{array}$ & 4 \\
\hline
\end{tabular}

Sumava has previously been shown to be very low in bulk density (group IIIB, with $m_{\infty} \approx 5000 \mathrm{~kg}$ ) while Benesov has been shown to be more like an ordinary chondrite, (group I with $m_{\infty} \approx 2000-4000 \mathrm{~kg}$ ). Svetsov (2000) also analyzed this bolide (assuming type Group II) and had determined that Sumava's behavior was inconsistent with the standard steady state, continuum theory, hydrodynamic flow solutions. In ReVelle (2004) the earlier group IIIB interpretation has been completely confirmed however.

Prompted by this extreme set of end heights, we reevaluated both Benesov and Sumava which are indicative of the two possible extreme compositions of observed fireballs. The mass loss prediction for Benesov in Figure 1 is extremely similar to that predicted by the approach of Ceplecha \& ReVelle (2005) from analyses of the observational flight data (see their Figure 28 for the analysis of the mass loss for Benesov for example). This agreement has also motivated us to reevaluate Revelstoke (Type II) in Carr (1970), Tagish Lake (Type II/IIIA) in Brown et al. (2002), Park Forest (Type I) in Brown et al. (2004), the 6/06/02 (Crete) bolide (Type I) in Brown et al. (2002) and the 9/03/04 Antarctic bolide (Type I/II) in Klekociuk et al. (2005).

\subsection{Additional Bolide Modeling Applications}

First we consider the Revelstoke meteorite fall over western Canada: 3/31/1965: It was a type II-body (with numerous infrasound recordings summarized in Bayer \& Jordan (1967) and in Shoemaker \& Lowery (1967), but with no light curve data) as reported in Folinsbee (1967), in Carr (1970) and in Shoemaker (1983) with one or possibly two very small carbonaceous chondrite meteorites having been recovered. In Figure 2, we have plotted the panchromatic luminosity, mass loss and $\sigma$ for Revelstoke. Shoemaker (1983) estimated the initial energy to be $\approx 20 \mathrm{kt}$ from the analysis of the infrasonic waves, but estimates by Edwards et al. $(2004,2006)$ are $\approx 20$ times smaller which is quite puzzling. Our Revelstoke entry modeling estimate resulted in an initial source energy $\approx 13.2 \mathrm{kt}$, but this value can't be constrained by a light curve since no data were available. 

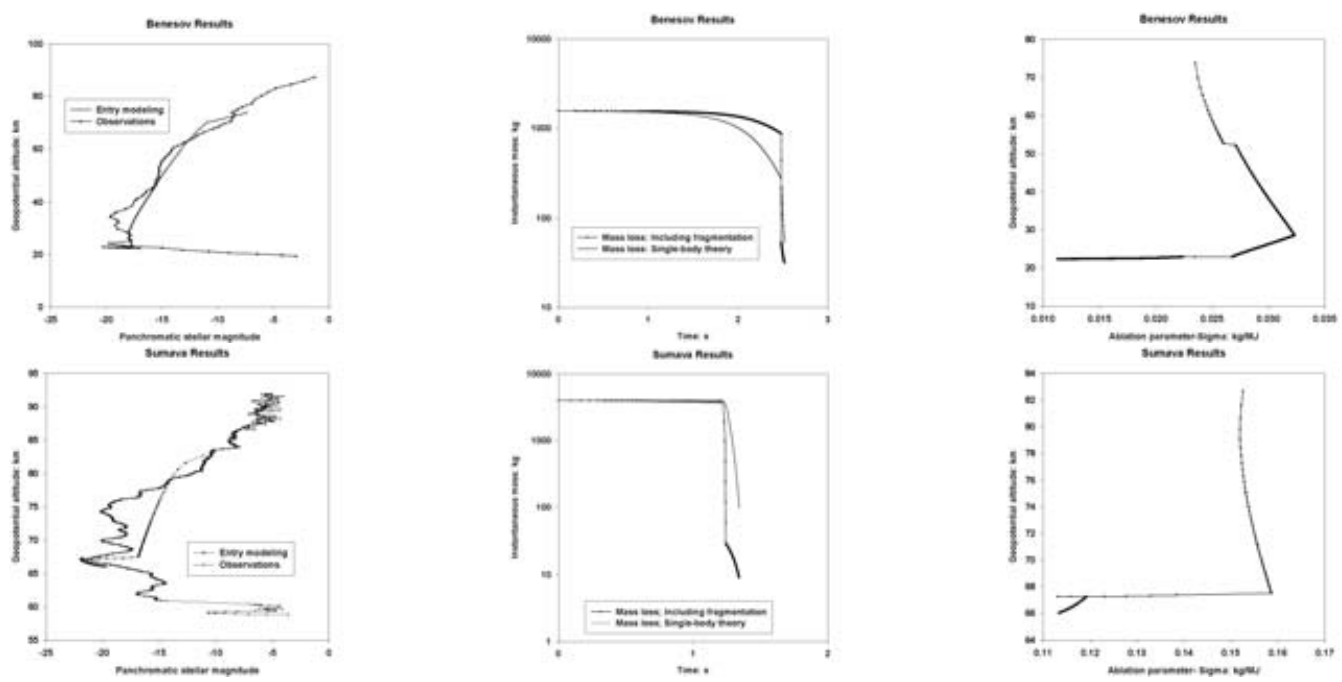

Figure 1. Top left: Benesov panchromatic light curve versus altitude: observed and modeled behavior; top center: Benesov mass loss: single-body and fragmentation modeling (see text); top right: Benesov ablation parameter $(\sigma)$ versus geopotential altitude; bottom left: Sumava panchromatic light curve versus altitude: observed and modeled behavior; bottom center: Sumava mass loss versus altitude: single-body and fragmentation modeling (see text); bottom right: Sumava ablation parameter $(\sigma)$ versus geopotential altitude.
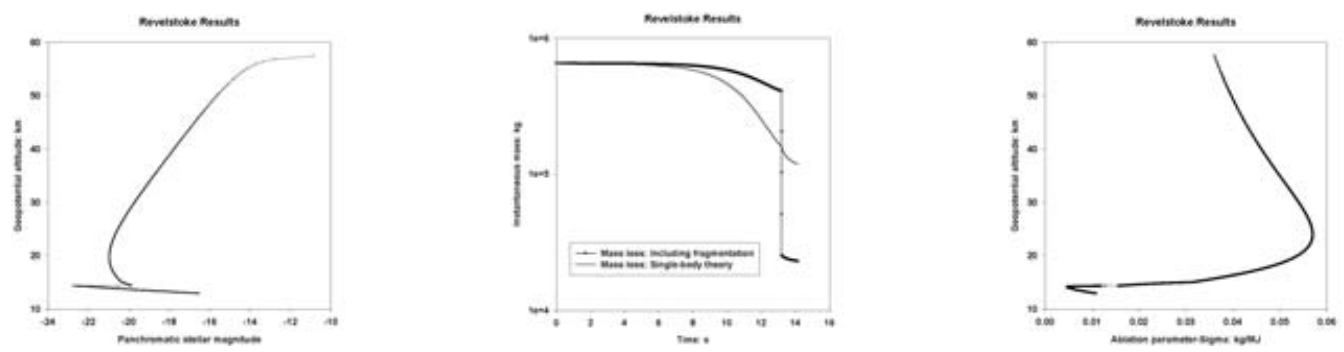

Figure 2. Left: Revelstoke panchromatic light curve versus altitude: observed and modeled behavior; center: Revelstoke mass loss versus altitude: single-body and fragmentation modeling (see text); right: Revelstoke ablation parameter $(\sigma)$ versus geopotential altitude.

Next we consider the Tagish Lake Bolide: 1/18/2000 (in the southern Canadian Yukon) - type II/IIIA with very large porosity noted in the recovered meteorites (with no infrasonic data currently available as discussed in Edwards et al. 2004, 2006). In Figure 3, we have plotted panchromatic luminosity, mass loss and $\sigma$ for Tagish Lake.

Next we modeled the Park Forest Meteorite Fall: 3/27/2003 (over Chicago) - type I with low porosity (with satellite detections, ground-based video and infrasonic detections as well as meteorites) given in Brown et al. (2004). In Figure 4, we have plotted the panchromatic luminosity, mass loss and $\sigma$ for Park Forest.

Next we considered the Crete Bolide: 6/06/2002: type I (with satellite detections and infrasound), in Brown et al. 2002. In Figure 5, we have plotted the behavior of the panchromatic luminosity, mass loss and $\sigma$ for the Crete bolide.

Finally, we considered the Antarctic bolide of 9/03/04 - type I/II bolide (with satellite detections and infrasound data available). In Figure 6 we have plotted the behavior of this small Aten asteroid (Klekociuk et al. 2005) for the panchromatic luminosity, the mass loss and $\sigma$. 

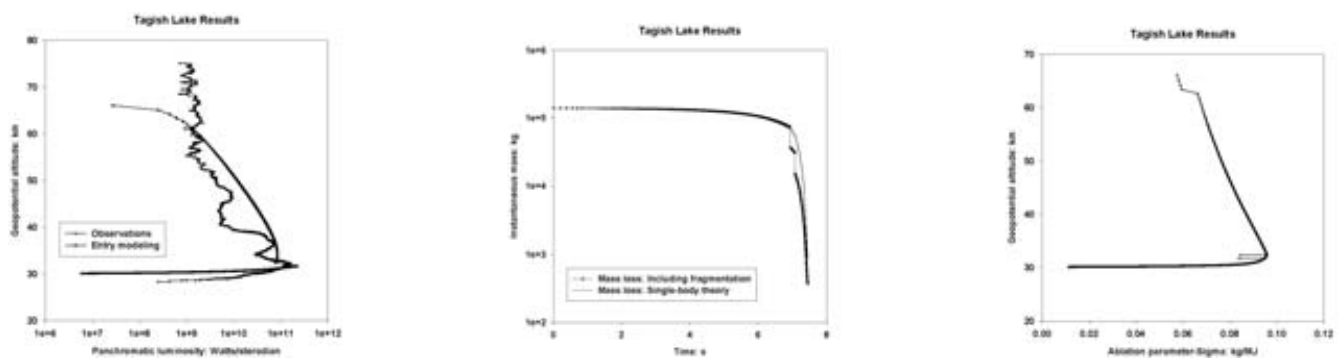

Figure 3. Left: Tagish Lake panchromatic light curve versus altitude: observed and modeled behavior; center: Tagish Lake mass loss versus altitude: single-body and fragmentation modeling (see text); right: Tagish Lake ablation parameter $(\sigma)$ versus geopotential altitude.
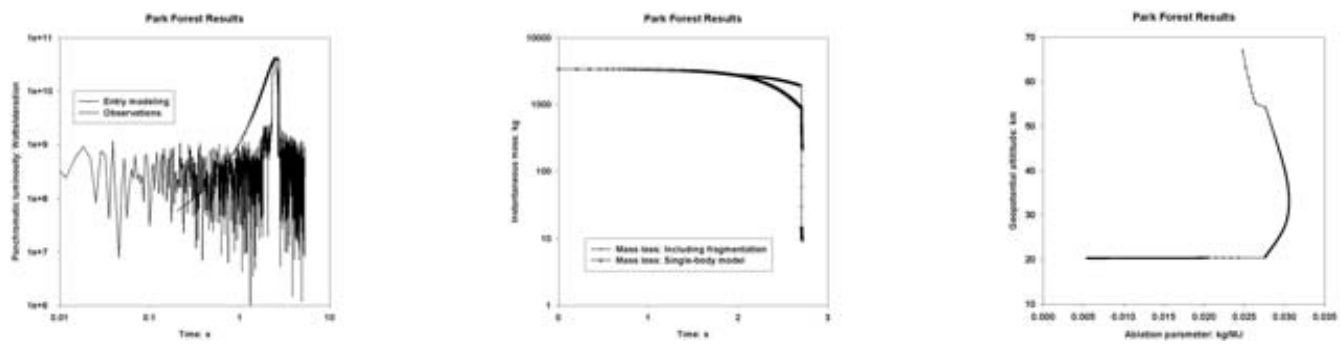

Figure 4. Left: Park Forest panchromatic light curve versus time: observed and modeled behavior; center: Park Forest mass loss versus time: single-body and fragmentation modeling (see text); right: Park Forest ablation parameter $(\sigma)$ versus geopotential altitude.
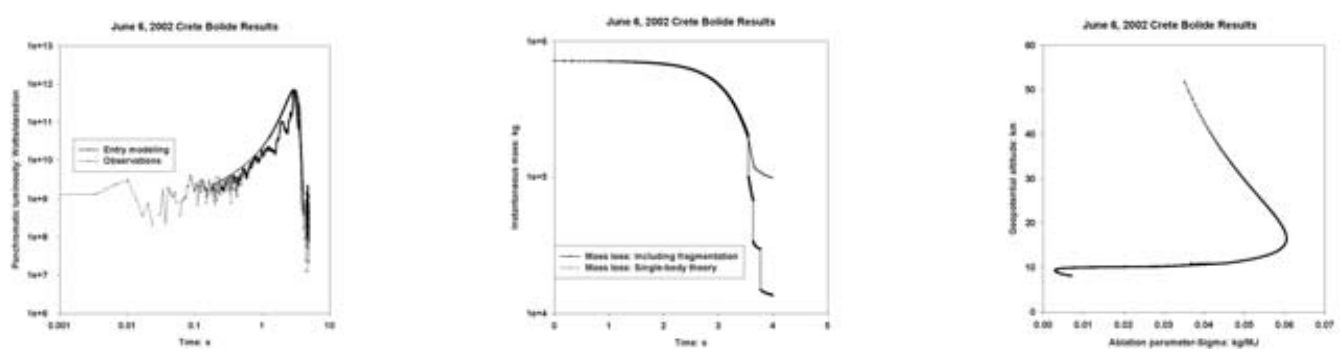

Figure 5. Left: Crete bolide panchromatic light curve versus time: observed and modeled behavior; center: Crete bolide mass loss versus time: single-body and fragmentation modeling (see text); right: Crete bolide ablation parameter $(\sigma)$ versus geopotential altitude.

One unusual circumstance for this event, based upon our modeling is that the "best fit" for the light curve has an extremely low end height (see the right panel og Figure 6), comparable to that of Great Siberian Meteor of 1908.

Overall the success of the agreement of our entry modeling code predictions with fireball observational data and its analysis seems to be rapidly converging.

\section{Summary and Conclusions}

\subsection{New Entry Modeling Technique}

In this paper we have introduced a new modeling technique to determine the terminal mass of a meteoroid during extensive fragmentation. It is based upon a new method for the prediction of the mass to area ratio which can be used to calculate the subsequent mass loss during entry quite reliably (compared to the single-body model predictions of 

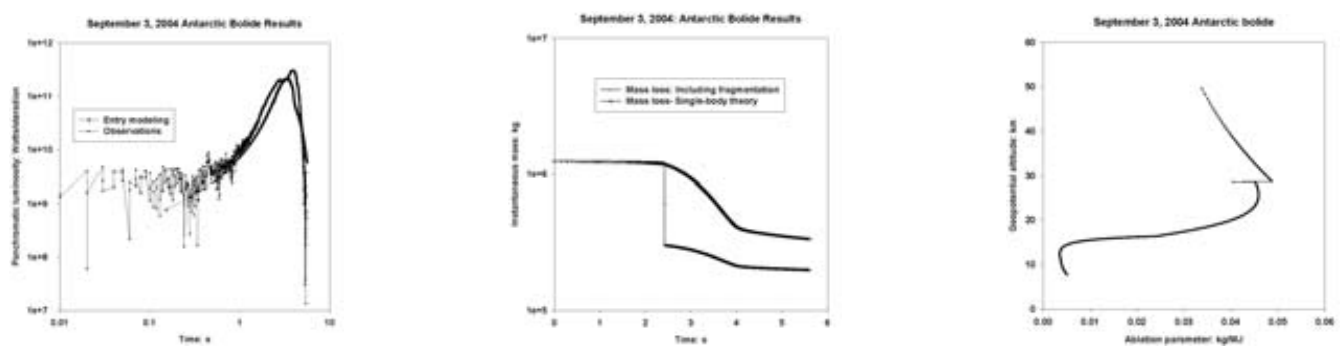

Figure 6. Left: Antarctic bolide: Power time curve (watts $/ \mathrm{m}^{2} /$ sterad) versus time: observed and modeled behavior; center: Antarctic bolide mass loss versus time: single-body and fragmentation modeling (see text); right: Antarctic bolide ablation parameter $(\sigma)$ versus geopotential altitude.

the past). We have compared the prediction of the single-body mass loss equation for each of the bolides against the new approach. In each case with extensive fragmentation, we have found that the single-body mass loss is an extreme upper limit to the "true" mass. Said another way, we have found that the mass loss calculated including fragmentation is generally significantly lower than that predicted using the single-body method. The predicted behavior is also very similar to the results presented in Ceplecha \& ReVelle (2005).

In addition, we have also found that our ablation parameter, $\sigma(z)$, is predicted to strongly decrease during fragmentation events which is also in excellent agreement with the systematic analyses of observations in Ceplecha \& ReVelle (2005). In fact, had we allowed fragmentation into a very large number of very small fragments instead of the simple factor of two reduction in mass allowed at each fragmentation point, the predicted $\sigma$ would have become even much smaller, in excellent agreement with Ceplecha \& ReVelle (2005). This strongly argues that many observed events have disintegrated into a large number of very small fragments so that the intrinsic $\sigma$ is at least an order of magnitude or more less than the apparent $\sigma$.

There is still much interesting physics to be incorporated into the bolide modeling process. Although results exist to correct the shock wave radiation flux to the body as a function of the "bluntness" of the body, the author has not yet incorporated this behavior as a function of the $\mu$ parameter, etc. Additional physics yet to incorporate also includes interference heating (shock wave/gas cap viscous boundary layer interaction at larger Knudsen number indicative of the transitional flow regime or generally for smaller bodies), ablation products absorption of the shock wave radiation flux, precursor ionization or free stream absorption effects produced by shock wave ultraviolet radiation (a preheating of the ambient air ahead of the body that modifies the "static" atmospheric density structure), etc. Still other effects include thermal conduction and melting of small fragmented particles, rotational effects on shape change and on light production, and finally the evaluation of lift forces, all need much further work for a greater understanding of this extremely complicated natural and wondrous phenomenon.

\subsection{Improved Bolide Initial Mass Estimates}

In addition, we have applied this new entry technique to a number of fireballs to evaluate its overall predictability for a diverse group of NEOs. The fireballs tested include not only meteorite-dropping events, but also highly friable bolides like Sumava, etc. This friable behavior also includes the Tagish Lake bolide whose detailed behavior includes remarkably the recovery of meteorites from an extremely low density and highly porous and friable body. Using our new mass loss technique, we have been able to determine more reliable terminal mass estimates for each of the seven fireballs examined. Our analyses 
have determined that Benesov (type I), Park Forest (type I), the Crete bolide of June 6, 2002 (type I) and finally the Antarctic bolide of September 3, 2004 (type I/II) are all indicative of stronger and denser bodies indicating the relative likelihood of a significant and recoverable meteorite fall. It is extremely remarkable that Tagish Lake is both extremely friable and yet produced a well documented meteorite fall (having impacted on a frozen lake). The particles that did not arrive as ponderable bodies on the earth must have been extremely friable and of very low density or else they were completely pulverized into extremely small bodies during entry (again in agreement with the very much reduced intrinsic $\sigma$ in comparison to the predicted apparent $\sigma$ ). The bulk porosity of Tagish Lake meteorite samples was about $45 \%$ (Brown et al. 2002) while our entry modeling results indicated the best agreement with the observed light curve at a porosity of $\approx 55-60 \%$ for the original body (with respect to an ordinary chondrite bulk density). This strongly reminds us that the entering bodies are highly inhomogeneous even if they are not significantly porous, a fact that the current author has been recently emphasizing (ReVelle 2004).

\section{Acknowledgements}

We would like to thank the Los Alamos GNEM program and the US DOE HQ in NA-22 for continued financial support.

\section{References}

Bayer, K.C. \& Jordan, J.N. 1967, J. Acoust. Soc. of America 41, 1580

Borovička, J. \& Spurný, P. 1996, Icarus 121, 484

Borovička, J., Popova, O.P., Nemtchinov, I.V., Spurný, P. \& Ceplecha, Z. 1998, Astron. Astrophys. 334, 713

Bronshten, V.A. 1983, Physics of Meteoric Phenomena (Dordrecht: Kluwer)

Brown, P.G., ReVelle, D.O., Tagliaferri E. \& Hildebrand, A.R. 2002, Meteorit. \& Planet. Sci. 37,661

Brown, P.G., Spalding, R.E., ReVelle, D.O., Tagliaferri, E. \& Worden, S.P. 2002, Nature 420, 314

Brown, P.G., Pack, D., Edwards, W.N., ReVelle, D.O., Yoo, B.B., Spalding, R.E. \& Tagliaferri, E. 2004, Meteorit. \& Planet. Sci. 39, 1781

Carr, M.H. 1970, Geochim. Cosmochim. Acta 34, 689

Ceplecha, Z., Borovička, J., Elford, W.G., ReVelle, D.O., Hawkes, R.L., Porubčan, V. \& Šimek, M. 1998, Space Sci. Rev. 84, 327

Ceplecha, Z. \& ReVelle, D.O. 2005, Meteorit. \& Planet. Sci. 40, 35

Edwards, W.N., Brown, P.G. \& ReVelle, D.O. 2004, Earth, Moon $\&$ Planets 95, 501

Edwards, W.N., Brown, P.G. \& ReVelle, D.O. 2006, J. Atmos. E Sol. Terrestr. Phys. 68, 1136

Folinsbee, R.E., Douglas, J.A.V. \& Maxwell, J.A. 1967, Geochim. Cosmochim. Acta 31, 1625

Klekociuk, A.R., Brown, P.G., Pack, D.W., ReVelle, D.O., Edwards, W.N., Spalding, R.E., Tagliaferri, E., Yoo, B.B. \& Zagari, J. 2005, Nature 436, 1132

ReVelle, D.O. 1979, J. Atmos. \& Terrestr. Phys. 41, 453

ReVelle, D.O. \& Ceplecha, Z. 2001, in: B. Warmbein (ed.) ,Meteoroids 2001, ESA Special Publ. 495 , p. 551

ReVelle, D.O. 2004, Earth, Moon $\&$ Planets 95, 441

Shoemaker, E.M. \& Lowery, C.J. 1967, J. Meteorit. Soc. 3, 123

Shoemaker, E.M. 1983, Annu. Rev. Earth E Planet. Sci. 11, 461

Svetsov, V.V. 2000, Sol. Syst. Research 34, 302 